\title{
Molecular features of colorectal polyps presenting Kudo's type II mucosal crypt pattern: are they based on the same mechanism of tumorigenesis?
}

Authors

Institutions
Kensuke Shinmura' ${ }^{1}$, Kazuo Konishi' ${ }^{1}$, Toshiko Yamochi ${ }^{2}$, Yutaro Kubota ${ }^{1}$, Yuichiro Yano ${ }^{1}$, Atsushi Katagiri', Takashi Muramoto', Toshihiro Kihara', Masayuki Tojo', Kenichi Konda', Teppei Tagawa', Fumito Yanagisawa', Mari Kogo ${ }^{3}$, Reiko Makino ${ }^{4}$, Masafumi Takimoto ${ }^{2}$, Hitoshi Yoshida ${ }^{1}$

Institutions are listed at the end of article. submitted 5. February 2014 accepted after revision 10. June 2014

\section{Bibliography}

Dol http://dx.doi.org/ 10.1055/s-0034-1377518

Published online: 18.7.2014

Endoscopy International Open 2014; 02: E171-E177

(c) Georg Thieme Verlag KG Stuttgart · New York E-ISSN 2196-9736

\section{Corresponding author}

\section{Kazuo Konishi, MD PhD}

Division of Gastroenterology,

Department of Medicine

Showa University School of

Medicine

1-5-8 Hatanodai, Shinagawa-ku

Tokyo 142-8666

Japan

Fax: +81-3-37847553

konishigi@hotmail.com
Background and study aims: The molecular features of serrated polyps (SPs) with hyperplastic crypt pattern, also called Kudo's type II observed by chromoendoscopy, were evaluated.

Methods: The clinicopathological and molecular features of 114 SPs with a hyperplastic pit pattern detected under chromoendoscopy (five dysplastic SPs, 63 sessile serrated adenoma/polyps (SSA/Ps), 36 microvesicular hyperplastic polyps (MVHPs), and 10 goblet cell-rich hyperplastic polyps (GCHPs)) were examined. The frequency of KRAS and $B R A F$ mutations and $\mathrm{CpG}$ island methylator phenotype (CIMP) were investigated.

Results: Dysplastic SPs and SSA/Ps were frequently located in the proximal colon compared to others (SSA/Ps vs. MVHPs or GCHPs, $P<0.0001$ ). No significant difference was found in the frequency of BRAF mutation among SPs apart from GCHP (60\% for dysplastic SPs, $44 \%$ for SSA/Ps, $47 \%$ for

\section{Introduction}

\section{$\checkmark$}

Colorectal cancer (CRC) is the third most common cancer in the world and the second leading cause of cancer-related deaths [1]. Most CRCs develop over a long period via a multistep process through the adenoma-carcinoma sequence [2]. About two-thirds of sporadic CRCs arise from conventional adenomas $[3,4]$. The process of colorectal carcinogenesis often begins with inactivation of the $A P C / \beta$-catenin signaling pathway, followed by KRAS and TP53 mutations. However, serrated adenomas (SAs), especially sessile serrated adenoma/polyps (SSA/Ps), have recently been described as immediate precursors for CRCs that develop via an alternative pathway with $\mathrm{CpG}$ island methylator phenotype (CIMP) and BRAF mutation [5]. In addition, CIMP cancer may develop via either a mutator (microsatellite instability) pathway or a pathway that leads to microsatellite stability [5]. This alternative pathway is called the serrated pathway.
MVHPs, and $0 \%$ for GCHPs). The frequency of CIMP was higher in dysplastic SPs or SSA/Ps than in MVHPs or GCHPs (60\% for dysplastic SPs, 56\% for SSA/Ps, 32\% for MVHPs, and 10\% for GCHPs) (SSA/Ps vs. GCHP, $P=0.0068$ ). When serrated neoplasias (SNs) and MVHPs were classified into proximal and distal lesions, the frequency of CIMP was significantly higher in the proximal compared to the distal SNs (64\% vs. $11 \%, P=$ 0.0032 ). Finally, multivariate analysis showed that proximal location and BRAF mutation were significantly associated with an increased risk of CIMP.

Conclusions: Distinct molecular features were observed between proximal and distal SPs with hyperplastic crypt pattern. Proximal MVHPs may develop more frequently through SSA/Ps to CIMP cancers than distal MVHPs.

Hyperplastic polyps (HPs) are often detected in the colorectum, especially in the sigmoid colon and rectum, of elderly patients $[6,7]$. For many years, HPs have been considered to be non-neoplastic lesions without malignant potential. Thus, they have been dismissed as innocuous. However, some HPs show molecular features similar to those of CRCs $[8,9]$. Recently, the serrated pathway has been proposed to begin with HPs, and then progress through SSA/Ps (with or without cytological dysplasia) to CRCs [5]. The serrated pathway appears to be involved in the formation of approximately $10 \%$ of CRCs [10].

Because of improvements in endoscopic imaging, details of the colorectal mucosa surface can be observed with colonoscopies. However, several studies $[11,12]$ have reported a low diagnostic accuracy of any of the novel endoscopic imaging techniques for differentiating SSAs from HPs. However, Kimura et al. [13] have proposed a type II open-shape pit pattern (Type II-O) which was specific to SSA/Ps. This crypt pattern was similar 
to the hyperplastic crypt pattern (stellar or papillary pits) but the pits were wider and more rounded in shape, reflecting dilatation of the crypts. However, their sensitivity and negative predictive value for the diagnosis of SSA/P were still insufficient. Our previous study [14] indicated that SAs occasionally showed a hyperplastic crypt pattern and that they presented distinct molecular features compared to SAs with a non-hyperplastic pit pattern. These are implicated in the therapeutic management of serrated polyps (SPs).

We hypothesized that biological or molecular differences exist among SPs with a hyperplastic crypt pattern, and that these features are associated with their different pathways of progression to CRCs. To test this hypothesis, the molecular features of SPs with a hyperplastic crypt pattern were investigated.

\section{Methods}

\section{Patients and samples}

In total, 4149 colorectal polyps were evaluated for their mucosal crypt patterns and then endoscopically resected at the Showa University Hospital between February 2009 and August 2012. Of these polyps, 318 revealed a hyperplastic pit pattern (Kudo's type II) under chromoendoscopy. These 318 polyps were examined and included 33 dysplastic SPs (10.4\%), 136 SSA/Ps (42.8\%), 102 HPs (32.1\%), 43 conventional adenomas (13.5\%), one inflammatory polyp $(0.3 \%)$, and three polyps without histological evaluation (0.9\%). Overall, 271 lesions were SPs (85\%, 271/318). Among the 271 SPs, we examined 114 SPs with a hyperplastic pit pattern (Kudo's type II) from 85 patients. The tissue samples were selected solely based on tissue availability. However, there were no significant differences in the clinicopathological features between overall and studied SP groups ( $\bullet$ Table S1).

Before SP samples were selected, patients with a) a familial predisposition to cancers such as familial adenomatous polyposis, hereditary nonpolyposis colorectal cancer or hyperplastic polyposis, or b) inflammatory bowel disease such as ulcerative colitis were excluded. The procedures for tissue collection and analysis were approved by the ethics committee of the Showa University School of Medicine. Written informed consent was obtained from each patient.

\section{Endoscopic evaluation}

All patients were prepared for the procedure with administration of $1.8 \mathrm{~L}$ of an oral electrolyte lavage solution. Colonoscopists with extensive experience performed all examinations using high definition colonoscopes (CF260AI; Olympus Optical Co., Tokyo, Japan) or high definition magnifying colonoscopes (PCF240Z or CF260HZI; Olympus Optical Co., Tokyo, Japan). The lesions found during colonoscopy were classified as polypoid or nonpolypoid on the basis of the Paris endoscopic classification [15]. We followed a procedure previously reported for the observation of mucosal crypt patterns on the surface of the lesion [16]. The mucosal crypt pattern of each lesion was determined according to the modified classification of Kudo et al. [17]. Briefly, Kudo's type II (stellar or papillary pits) mucosal crypt patterns were defined as a hyperplastic pattern ( $\bullet$ Fig. $\mathbf{1} \mathbf{b}$ ). If the lesion revealed this pit pattern, we considered whether it should be included in this study. However, the lesion was excluded from this study when it presented a mixed pattern comprised of a combination of a hyperplastic pattern and other patterns.

SSA/Ps are typically $10 \mathrm{~mm}$ or greater in size, located in the proximal colon and covered with a mucus cap $(\bullet$ Fig. 1 a). Their mucosal crypt pattern is usually the Kudo's type II pit pattern, which is similar to the hyperplastic polyp. Thus, the following criteria were used to decide whether lesions that exhibited a hyperplastic pattern on their surface should be removed endoscopically: (i) typical location of HPs in the rectosigmoid area, and (ii) small size (usually $\leq 5 \mathrm{~mm}$ in diameter) and a symmetrical and uniform shape. If a colonoscopist established a diagnosis of typical HP, no further endoscopic treatment was performed. Otherwise, lesions were removed using coagulation-biopsy ("hot biopsy") or endoscopic resection (i.e., endoscopic mucosal resection or polypectomy).

\section{Tissue samples and histological evaluation}

Serial sections $(3 \mu \mathrm{m})$ were obtained from paraffin blocks and prepared for hematoxylin and eosin (H\&E) staining. All H\&Estained slides were reviewed by a senior pathologist (TY) who was blinded to the endoscopic findings. SPs were classified into HP, SSA/P, or traditional serrated adenoma (TSA) on the basis of the WHO classification [18] ( $\bullet$ Fig. 2). A SSA/P accompanied by a cytologically dysplastic area was defined as a SSA/P with cytological dysplasia (SSA/P-cd) ( $\bullet$ Fig. 2b). In the present study, dys-

TableS1 Comparison of clinicopathological features between overall and studied serrated polyps.

\begin{tabular}{|c|c|c|c|c|c|c|c|}
\hline & & \multicolumn{3}{|c|}{$\begin{array}{l}\text { Overall SP samples } \\
(\mathrm{n}=\mathbf{2 7 1})\end{array}$} & \multicolumn{3}{|c|}{$\begin{array}{l}\text { Studied SP samples } \\
(n=114)\end{array}$} \\
\hline & & $\begin{array}{l}\text { Dysplastic SP } \\
(\mathrm{n}=33)\end{array}$ & $\begin{array}{l}\text { SSA } / P \\
(n=136)\end{array}$ & $\begin{array}{l}\text { HP } \\
(n=102)\end{array}$ & $\begin{array}{l}\text { Dysplastic SP } \\
(n=5)\end{array}$ & $\begin{array}{l}S S A / P \\
(n=63)\end{array}$ & $\begin{array}{l}\text { HP } \\
(n=46)\end{array}$ \\
\hline \multirow[t]{2}{*}{ Gender } & Male & $18(55 \%)$ & $79(58 \%)$ & $71(70 \%)$ & $3(60 \%)$ & $38(60 \%)$ & $33(72 \%)$ \\
\hline & Female & $15(45 \%)$ & $57(42 \%)$ & $31(30 \%)$ & $2(40 \%)$ & $25(40 \%)$ & $13(28 \%)$ \\
\hline \multirow[t]{2}{*}{ Age } & (Median, years) & 62 & 60 & 59 & 54 & 61 & 63 \\
\hline & (Range, years) & $23-82$ & $32-84$ & $35-82$ & $23-69$ & $33-79$ & $35-80$ \\
\hline \multirow[t]{2}{*}{ Tumor location } & Proximal & $19(58 \%)$ & $108(79 \%)$ & $52(51 \%)$ & $4(80 \%)$ & $55(87 \%)$ & $17(37 \%)$ \\
\hline & Distal & $14(42 \%)$ & $28(21 \%)$ & $50(49 \%)$ & $1(20 \%)$ & $8(13 \%)$ & $29(63 \%)$ \\
\hline \multirow[t]{2}{*}{ Size } & (Median, mm) & 8 & 10 & 7 & 8 & 10 & 7 \\
\hline & (Range, mm) & $5-45$ & $4-25$ & $3-20$ & $7-12$ & $5-23$ & $4-11$ \\
\hline \multirow[t]{3}{*}{ Macroscopic type } & Protruded & $7(21 \%)$ & 0 & $13(13 \%)$ & 0 & $1(2 \%)$ & $7(15 \%)$ \\
\hline & Superficial & $26(79 \%)$ & $135(99 \%)$ & $88(86 \%)$ & $5(100 \%)$ & $61(96 \%)$ & $37(81 \%)$ \\
\hline & Combined & 0 & $1(1 \%)$ & $1(1 \%)$ & 0 & $1(2 \%)$ & $2(4 \%)$ \\
\hline
\end{tabular}

Dysplastic SPs include traditional serrated adenoma and SSA/P with cytological dysplasia. SP, serrated polyp; SSA/P, sessile serrated adenoma/polyp; HP, hyperplastic polyp. 

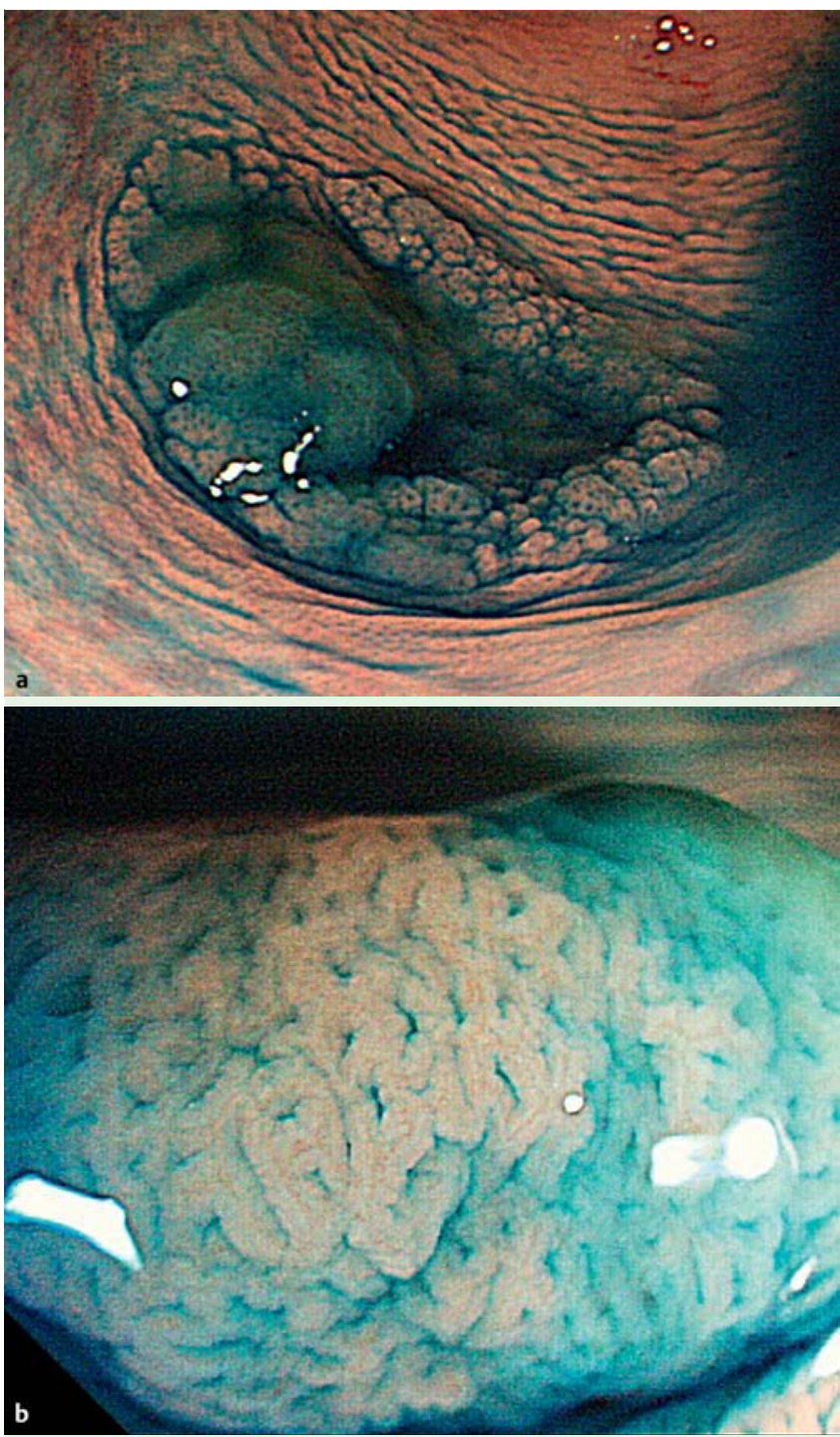

Fig. 1 Endoscopic appearance of serrated polyps (all lesions were observed after spraying with indigo carmine dye): a Ordinary view of sessile serrated adenoma/polyp; b Magnifying view of hyperplastic pit pattern (Kudo's type II) on the surface of the polyp by magnifying chromoendoscopy.

plastic SPs and SSA/Ps were defined as serrated neoplasias (SNs). Moreover, HPs were divided into three subtypes, namely, the microvesicular hyperplastic polyp (MVHP) $(\boldsymbol{O}$ Fig.2c), the goblet cell-rich hyperplastic polyp (GCHP) ( $\bullet$ Fig.2d), and the mucinpoor hyperplastic polyp on the basis of the WHO classification [18].

Genomic DNA was extracted from 45 formalin-fixed, paraffinembedded samples and 69 frozen tissue samples. The frozen tissue samples were obtained from the lesions using colonoscopic biopsy before endoscopic resection and were stored at $-80^{\circ} \mathrm{C}$. We distinguished between tumor and adjacent colonic mucosa based on pit patterns observed by chromoendoscopy. DNA was extracted from the frozen tissue samples using the standard proteinase $\mathrm{K} / \mathrm{phenol} / \mathrm{chloroform}$ method. As for formalin-fixed, paraffin-embedded samples, serial slides were obtained from the archival blocks of formalin-fixed, paraffin-embedded tumor tissues, which were resected endoscopically. One slide was stained with H\&E for microdissection. After microdissection, DNA was extracted using the QIAamp DNA mini kit (QIAGEN Inc., Valencia, CA).
Bisulfite polymerase chain reaction (PCR) and pyrosequencing analysis of DNA methylation

Bisulfite treatment was performed as previously described [19]. Bisulfite-treated DNA (2 or $3 \mu \mathrm{L}$ ) was used as the template for PCR. The primers and PCR conditions used for the amplification of specific DNA fragments of various target genes were set according to those described in our previous report [20]. The protocol for pyrosequencing, a quantitative tool for methylation density, was previously described in detail [20].

\section{Methylation-related genes and definition of the CpG island methylator phenotype (CIMP)}

It has been reported that sporadic CRCs can be classified into two groups: CIMP-positive and CIMP-negative according to the frequency of methylation of the $\mathrm{CpG}$ islands in the promoter of five genes (MINT1, MINT2, MINT31, CDKN2A (p16), and MLH1) [21]. The determination of CIMP status required a quantitative tool, and methylation positivity was defined as a methylation density greater than $15 \%$. A tumor was considered to be CIMP-positive if two or more of the CIMP markers were methylated as previously described [20]. All others were defined as CIMP-negative.

\section{KRAS and BRAF mutations}

PCR-based pyrosequencing assays were used to analyze samples for the presence of activating mutations in codons 12 and 13 of KRAS and in codon 600 of BRAF, as previously described [22].

\section{Data analysis and statistics}

We analyzed 114 SPs as follows: 1) clinicopathological and molecular features were investigated by histological findings or by tumor location, and 2) the relationship between CIMP and clinicopathological/molecular features was evaluated. As for the latter analysis, we first examined the relationship between CIMP-positive SPs and gender, age, tumor location (proximal vs. distal), tumor size, macroscopic type (protruded vs. superficial, elevated), histology (SN vs. HP), or genetic alterations (BRAF and KRAS mutations) by univariate analysis. In this analysis, gender, tumor location, macroscopic type, histology, and genetic alterations were considered to be categorical variables, whereas age and tumor size were continuous variables. Next, logistic regression analysis was used to calculate the relationship between CIMP-positive SPs and significant factors that were selected by univariate analysis. Continuous variables (i.e., age and tumor size) were analyzed using the Wilcoxon test. Categorical variables were compared between tumor groups using the $\mathrm{X}^{2}$ test or Fisher's exact test when testing small samples. The odds ratio (OR) and 95\% confidence interval $(\mathrm{CI})$ were determined for a variety of factors. All tests were two-sided, and $P<0.05$ was considered statistically significant. However, when multiple comparisons were performed, $P$ values were considered to be significant at a Bonferroni-corrected alpha of $0.05 / 6$ for four groups. All statistical analyses were performed with SAS version 9.2 and JMP version 10 (SAS Institute, Inc., Cary, NC). 

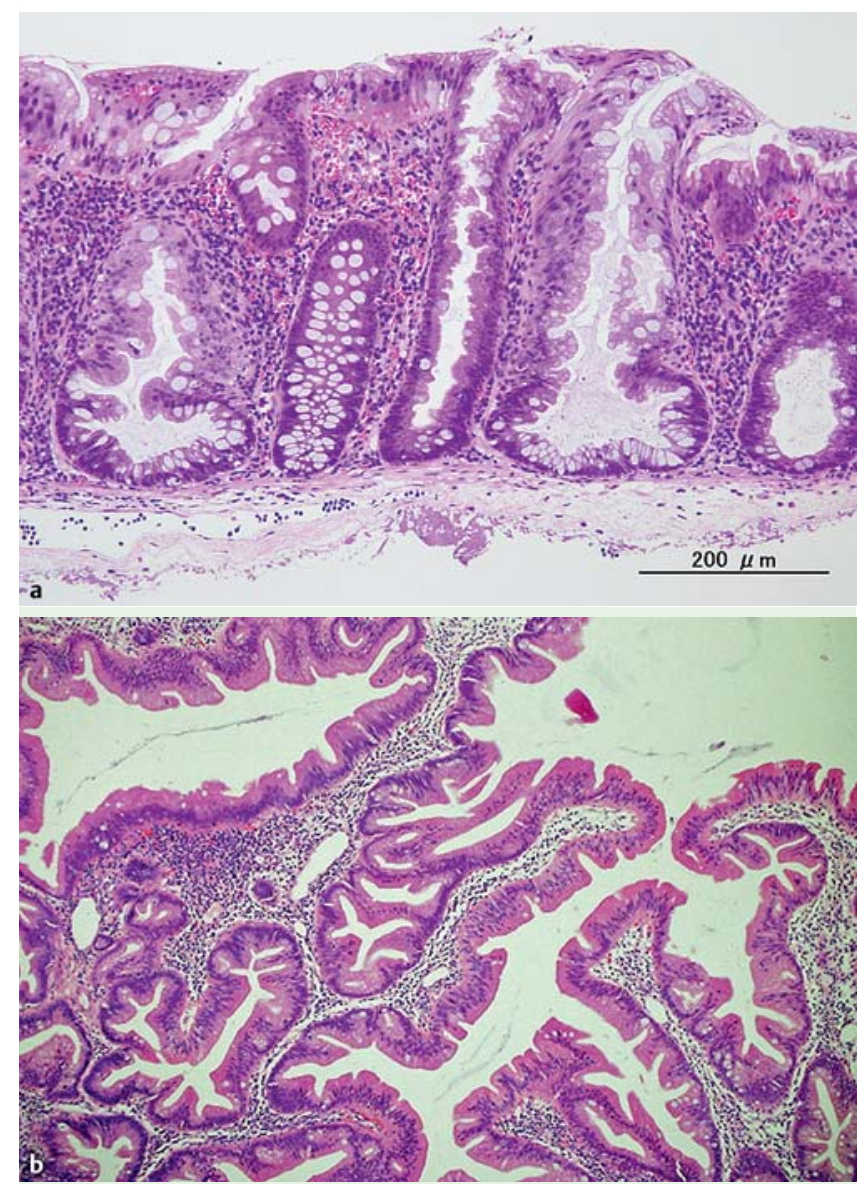

Fig. 2 Histopathology of serrated polyps: a Sessile serrated adenoma/polyp (SSA/P). The crypts with serration are distorted and dilated, L-shaped, inverted T-shaped or anchor-shaped; b SSA/P with cytological dysplasia. The cytological dysplasia in SSA/P is histologically different from conventional adenoma. The nuclei have more open chromatin and prominent nucleoli. The cytoplasm is more eosinophilic than that observed in conventional adenoma; c Microvesicular hyperplastic polyps; $\mathbf{d}$ Goblet cell-rich hyperplastic polyps.

\section{Results}

$\nabla$

Comparison of clinicopathological and molecular features among SPs with type II pit pattern

The 114 SPs were histologically classified into five dysplastic SPs (four SSA/P-cd and one TSA), 63 SSA/Ps, 36 MVHPs and 10 GCHPs. - Table 1 shows the clinicopathological and molecular features of these lesions. Compared to MVHPs and GCHPs, dysplastic SPs and SSA/Ps were found more frequently in the proximal colon. The differences between SSA/Ps and MVHPs or GCHPs were statistically significant $(P<0.0001)$. Regarding lesion size, dysplastic SPs and SSA/Ps were larger than MVHPs or GCHPs (SSA/Ps vs. MVHPs, $P<0.0001$; SSA/Ps vs. GCHPs, $P=0.0005$ ). Macroscopically, nonpolypoid configuration (superficial, elevated type) was more frequently found in dysplastic SPs and SSA/Ps than in MVHPs or GCHPs. There were significant differences between SSA/Ps and GCHPs $(P=0.0068)$. The frequency of the BRAF mutation was significantly higher in dysplastic SPs, SSA/Ps or MVHPs than in GCHPs $(P<0.008)$. A higher frequency of CIMP was observed in dysplastic SPs and SSA/Ps than in MVHPs or GCHPs. The difference between SSA/Ps and GCHPs was statistically significant $(P=0.0068)$.
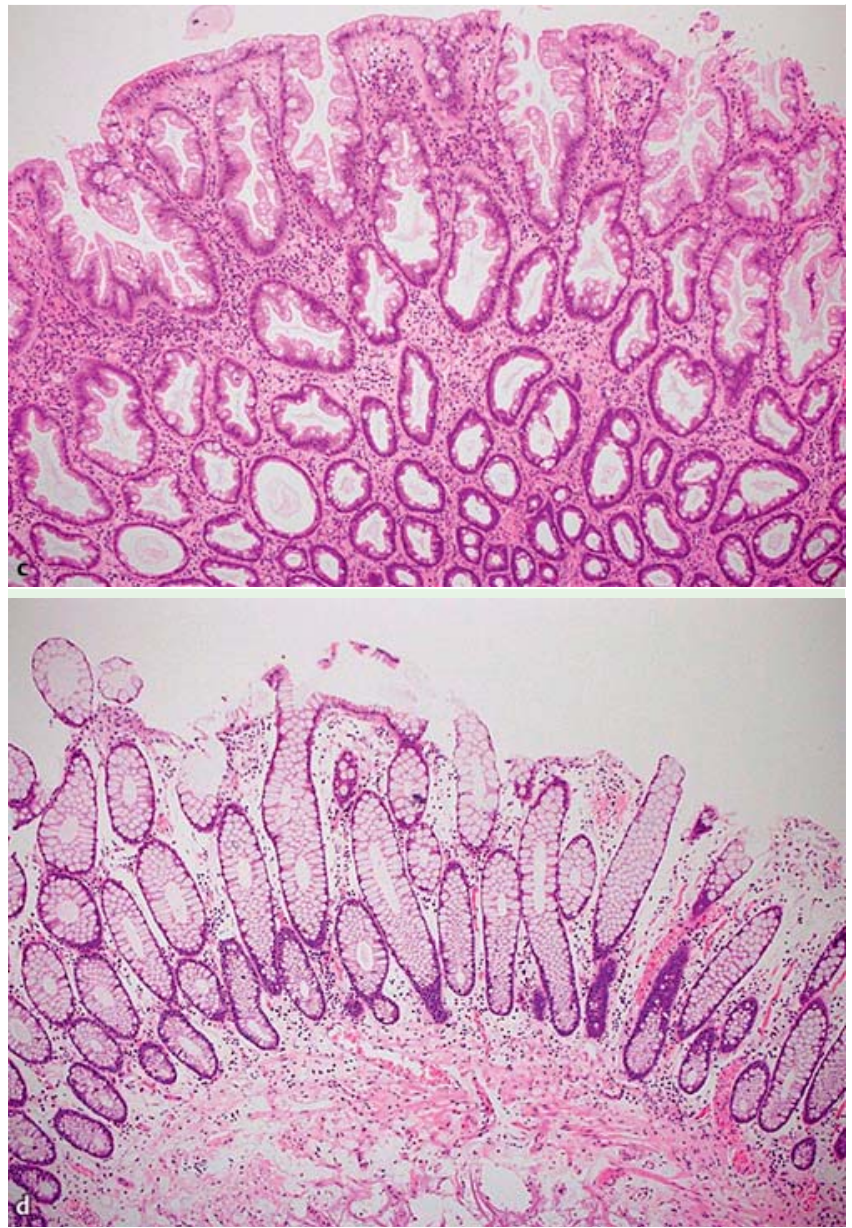

Comparison of clinicopathological and molecular features between proximal and distal SPs with type II pit pattern GCHPs were excluded from this analysis since significant differences were observed in the molecular features between GCHPs and others. As shown in $\odot$ Table2, proximal SNs were significantly larger than proximal MVHPs $(P<0.0001)$. Moreover, a significant difference in the frequency of CIMP was observed between proximal and distal SNs (64\% vs. $11 \%, P=0.0032$ ). Although the frequency of CIMP was higher in the proximal than in the distal MVHPs, this difference was not statistically significant ( $50 \%$ vs. $23 \%, P=0.0952$ ).

\section{Factors related to CIMP in SPs with type II pit pattern}

In the univariate analysis, five significant factors related to CIMP (tumor location, tumor size, macroscopic type, histology, and $B R A F$ mutation) were selected. Finally, logistic regression analysis revealed that proximal location as well as BRAF mutation was significantly associated with an increased risk of CIMP ( $\bullet$ Table 3$)$.

\section{Discussion}

$\nabla$

SAs, especially SSA/Ps, are thought to be precursor lesions of CIMP-positive CRCs [5]. The association of molecular alterations with the histologic subtypes of SPs and CIMP CRCs has led to the description of the sequential pathway from HP through SSA/P with or without cytological dysplasia to CIMP CRC [5]. This se- 


\begin{tabular}{|c|c|c|c|c|c|}
\hline & & $\begin{array}{l}\text { Dysplastic SP1 } \\
(n=5)\end{array}$ & $\begin{array}{l}S S A / P^{2} \\
(n=63)\end{array}$ & $\begin{array}{l}\text { MVHP } \\
(n=36)\end{array}$ & $\begin{array}{l}\text { GCHP } \\
(n=10)\end{array}$ \\
\hline \multirow[t]{2}{*}{ Gender } & Male & $3(60 \%)$ & $38(60 \%)$ & $28(78 \%)$ & $5(50 \%)$ \\
\hline & Female & $2(40 \%)$ & $25(40 \%)$ & $8(22 \%)$ & $5(50 \%)$ \\
\hline \multirow[t]{2}{*}{ Age } & (Median, years) & 54 & 61 & 63 & 64 \\
\hline & (Range, years) & $23-69$ & $33-79$ & $35-80$ & $49-77$ \\
\hline \multirow[t]{2}{*}{ Tumor location ${ }^{3}$} & Proximal & $4(80 \%)$ & $55(87 \%)$ & $14(39 \%)$ & $3(30 \%)$ \\
\hline & Distal & $1(20 \%)$ & $8(13 \%)$ & $22(61 \%)$ & $7(70 \%)$ \\
\hline \multirow[t]{2}{*}{ Size $^{4}$} & (Median, mm) & 8 & 10 & 6.5 & 6.5 \\
\hline & (Range, mm) & $7-12$ & $5-23$ & $4-11$ & $5-10$ \\
\hline \multirow{3}{*}{$\begin{array}{l}\text { Macroscopic } \\
\text { type }^{5}\end{array}$} & Protruded & 0 & $1(2 \%)$ & $5(14 \%)$ & $2(20 \%)$ \\
\hline & Superficial & $5(100 \%)$ & $61(96 \%)$ & $29(81 \%)$ & $8(80 \%)$ \\
\hline & Combined & 0 & $1(2 \%)$ & $2(5 \%)$ & 0 \\
\hline \multirow[t]{2}{*}{$B R A F^{6}$} & Mut+ & $3(60 \%)$ & $28(44 \%)$ & $17(47 \%)$ & 0 \\
\hline & Mut- & $2(40 \%)$ & $35(56 \%)$ & $19(53 \%)$ & $10(100 \%)$ \\
\hline \multirow[t]{2}{*}{ KRAS } & Mut+ & 0 & $6(10 \%)$ & $3(8 \%)$ & $3(30 \%)$ \\
\hline & Mut- & $5(100 \%)$ & 57 (90\%) & $33(92 \%)$ & $7(70 \%)$ \\
\hline \multirow[t]{2}{*}{$\mathrm{CIMP}^{7}$} & Presence & $3(60 \%)$ & $35(56 \%)$ & $12(33 \%)$ & $1(10 \%)$ \\
\hline & Absence & $2(40 \%)$ & 27 (44\%) & $24(67 \%)$ & $9(90 \%)$ \\
\hline
\end{tabular}

Table 1 Clinicopathological characteristics of serrated polyps with type II pit pattern.

SP, serrated polyp; SSA/P, sessile serrated adenoma/polyp; HP, hyperplastic polyp; MVHP, microvesicular HP; GCHP, goblet-cell rich HP; Mut+, presence of mutation; Mut-, absence of mutation.

1 Dysplastic SPs include traditional serrated adenoma and SSA/P with cytological dysplasia.

2 One SSA/P could not be determined CIMP status.

${ }^{3}$ SSA/P vs. MVHP or GCHP, $P<0.0001$.

${ }^{4} \mathrm{SSA} / \mathrm{P}$ vs. MVHP or GCHP, $P<0.0001$ or $P=0.0005$.

${ }^{5} \mathrm{SSA} / \mathrm{P}$ vs. GCHP, $P=0.0068$.

${ }^{6}$ GCHP vs. TSA, SSA $/ P$ or MVHP, $P=0.0082, P=0.0077$ or $P=0.0068$.

${ }^{7}$ GCHP vs. SSA $/ P, P=0.0068$.

\begin{tabular}{|c|c|c|c|c|c|}
\hline & & \multicolumn{2}{|l|}{ Proximal } & \multicolumn{2}{|l|}{ Distal } \\
\hline & & $\begin{array}{l}\mathrm{SN}^{1} \\
(n=59)\end{array}$ & $\begin{array}{l}\text { MVHP } \\
(n=14)\end{array}$ & $\begin{array}{l}\text { SN } \\
(n=9)\end{array}$ & $\begin{array}{l}\text { MVHP } \\
(n=22)\end{array}$ \\
\hline \multirow[t]{2}{*}{ Gender } & Male & $37(63 \%)$ & $9(64 \%)$ & $4(44 \%)$ & $19(86 \%)$ \\
\hline & Female & $22(37 \%)$ & $5(36 \%)$ & $5(56 \%)$ & $3(14 \%)$ \\
\hline \multirow[t]{2}{*}{ Age } & (Median, years) & 61 & 71.5 & 51 & 60 \\
\hline & (Range, years) & $23-79$ & $50-80$ & $33-76$ & $35-78$ \\
\hline \multirow[t]{2}{*}{ Size $^{2}$} & (Median, mm) & 10 & 5.5 & 9.5 & 7 \\
\hline & (Range, mm) & $5-23$ & $4-10$ & $5-12$ & $4-11$ \\
\hline \multirow[t]{3}{*}{ Macroscopic type } & Protruded & $1(2 \%)$ & 0 & 0 & $5(23 \%)$ \\
\hline & Superficial & 57 (96\%) & $13(93 \%)$ & $9(100 \%)$ & $16(73 \%)$ \\
\hline & Combined & $1(2 \%)$ & $1(7 \%)$ & 0 & $1(4 \%)$ \\
\hline \multirow[t]{2}{*}{ BRAF } & Mut + & $27(46 \%)$ & $4(29 \%)$ & $4(44 \%)$ & $13(59 \%)$ \\
\hline & Mut- & $32(54 \%)$ & $10(71 \%)$ & $5(56 \%)$ & $9(41 \%)$ \\
\hline \multirow[t]{2}{*}{ KRAS } & Mut + & $5(8 \%)$ & $1(7 \%)$ & $1(11 \%)$ & $2(9 \%)$ \\
\hline & Mut- & $54(92 \%)$ & $13(93 \%)$ & $8(89 \%)$ & 20 (91\%) \\
\hline \multirow[t]{2}{*}{$\mathrm{CIMP}^{3}$} & Presence & $37(64 \%)$ & $7(50 \%)$ & $1(11 \%)$ & $5(23 \%)$ \\
\hline & Absence & $21(36 \%)$ & $7(50 \%)$ & $8(89 \%)$ & $17(77 \%)$ \\
\hline
\end{tabular}

SN, serrated neoplasia; MVHP, microvesicular hyperplastic polyp; Mut+, presence of mutation; Mut-, absence of mutation.

${ }^{1}$ One SSA/P where CIMP status could not be determined.

2 Proximal SN vs. proximal MVHP, $P<0.0001$.

3 Proximal SN vs. distal SN, $P=0.0032$.

quence occurs more often in the proximal than in the distal colon. However, it remains uncertain whether SSA/Ps could develop directly from HPs or normal colon mucosa. Our data are in agreement with Buda et al. [23] who reported no significant differences in the frequency of BRAF mutations between proximal and distal SNs or MVHPs. Moreover, our data indicated that proximal MVHPs and SSA/Ps presented higher frequencies of CIMP than distal lesions. These observations suggest that proximal MVHPs could progress through SSA/Ps along with CIMP.

TSAs are more likely to be more molecularly diverse than SSA/Ps in that they might show either KRAS or BRAF mutations, or either low or high levels of CIMP $[5,24]$. In our study, only one TSA with a type II pit pattern had BRAF mutations and CIMP (data not shown). We previously reported a higher frequency of $B R A F$ mutations and CIMP in TSAs with a hyperplastic pit pattern, compared to those with other pit patterns [14]. Molecular diversity might be associated with mucosal crypt pattern on the surface of TSAs.

The current issue is considering the types of SP that should be removed endoscopically in clinical practice. However, several endoscopic features have recently been identified. SSA/Ps, especially in the proximal colon, are often covered with a tenacious mucus cap [25]. A recent prospective study reported that $64 \%$ of SSA/Ps presented a mucus cap [26]. Furthermore, a rim of debris 


\begin{tabular}{|c|c|c|c|c|c|c|}
\hline & & \multicolumn{2}{|l|}{ CIMP } & \multirow{2}{*}{$\begin{array}{l}\text { Univariate } \\
P \text { value }\end{array}$} & \multirow{2}{*}{$\begin{array}{l}\text { Multivariate } \\
\text { Odds ratio }(95 \% \mathrm{Cl})\end{array}$} & \multirow[b]{2}{*}{$P$ value } \\
\hline & & $\begin{array}{l}\text { Presence } \\
(n=51)\end{array}$ & $\begin{array}{l}\text { Absence } \\
(n=62)\end{array}$ & & & \\
\hline \multirow[t]{2}{*}{ Gender } & Male & $34(67 \%)$ & $39(63 \%)$ & 0.698 & & \\
\hline & Female & $17(33 \%)$ & $23(37 \%)$ & & & \\
\hline Age & (years) & 62.2 & 59.6 & 0.242 & & \\
\hline \multirow[t]{2}{*}{ Tumor location } & Proximal & $45(88 \%)$ & $30(48 \%)$ & $<0.001$ & $18.6(5.1-68.3)$ & $<0.001$ \\
\hline & Distal & $6(12 \%)$ & $32(52 \%)$ & & & \\
\hline Size & $(\mathrm{mm})$ & 10.6 & 8.4 & 0.006 & & \\
\hline \multirow[t]{2}{*}{ Macroscopic type } & Protruded ${ }^{1}$ & $11(22 \%)$ & $25(40 \%)$ & 0.043 & & \\
\hline & Superficial & $40(78 \%)$ & $37(60 \%)$ & & & \\
\hline \multirow[t]{2}{*}{ Histology } & $\mathrm{SN}^{2}$ & $38(75 \%)$ & $29(47 \%)$ & 0.004 & & \\
\hline & HP & $13(25 \%)$ & $33(53 \%)$ & & & \\
\hline \multirow[t]{2}{*}{ BRAF } & Mut+ & $33(65 \%)$ & $15(24 \%)$ & $<0.001$ & $13.0(4.1-41.3)$ & $<0.001$ \\
\hline & Mut- & $18(35 \%)$ & 47 (76\%) & & & \\
\hline \multirow[t]{2}{*}{ KRAS } & Mut + & $5(10 \%)$ & $7(11 \%)$ & $>0.999$ & & \\
\hline & Mut- & $46(90 \%)$ & $55(89 \%)$ & & & \\
\hline
\end{tabular}

Table 3 Clinicopathological and molecular factors associated with CIMP-positive serrated polyps

CIMP, CpG island methylator phenotype; SN, serrated neoplasia; HP, hyperplastic polyp; Mut+, presence of mutation; Mut-, absence of mutation.

1 Protruded type lesions include three combined type lesions.

${ }^{2}$ One SSA/P, where CIMP status could not be determined, was excluded from this analysis.

and bubbles, alteration of the contour of a fold and interruption of the underlying vascular pattern were identified as endoscopic features of SSA/Ps but not frequently found [26]. A dye-spraying or image-enhancing technique may allow for a reliable differentiation of SSA/Ps from HPs during colonoscopy. A type II-O pit pattern observed on the surface of the lesion is specific to SSA/P [13]. However, this is not sensitive for SSA/P (lower sensitivity and negative predictive value) [13]. Moreover, magnifying colonoscopy, which is rarely available in western countries, is necessary to recognize the type II-O pit pattern [25]. We previously reported that Kudo's type II pit pattern can be observed on the surface of SPs using non-magnifying colonoscopy [14].

Although our study was retrospective, several issues of therapeutic strategy for SPs have been addressed. Rex et al. [25] recommended the complete endoscopic removal of all SPs, apart from diminutive sigmoid or rectal lesions. Our criteria used to select the treatment of SPs with hyperplastic pattern were similar to their recommendations. In our study, $85.3 \%$ of polyps with a type II pit pattern, which were removed endoscopically in this study, showed serrated histology (SSA/P, TSA, and HP). However, the precursor polyp of CIMP CRCs needs to be removed effectively. Our logistic regression analysis indicated that proximal location as well as BRAF mutation were independent risk factors for SPs with CIMP. Therefore, endoscopists should aggressively remove proximal SPs when SPs with a type-II pit pattern measuring $6 \mathrm{~mm}$ or larger in size are detected by chromoendoscopy or electronic chromoendoscopy (e.g., narrow-band imaging).

In summary, our results indicate that proximal and distal SPs with a hyperplastic crypt pattern have distinctive molecular features and that proximal MVHPs may develop more frequently through SSA/Ps with or without cytological dysplasia to CIMP CRCs, compared to distal MVHPs. The appropriate screening and therapeutic intervention programs for CRCs should take these observations into account.

Competing interests: None
Institutions

${ }^{1}$ Division of Gastroenterology, Department of Medicine, Showa University School of Medicine, Tokyo, Japan

2 Department of Pathology, Showa University School of Medicine, Tokyo, Japan ${ }^{3}$ Department of Hospital Pharmaceutics, Showa University School of Pharmacy, Tokyo, Japan

${ }^{4}$ Clinical Collaborating Laboratory, Showa University School of Medicine, Tokyo, Japan

\section{Acknowledgement}

This work was supported in part by

JSPS KAKENHI grant\#24591029.

\section{References}

1 Ferlay J, Shin HR, Bray F et al. Estimates of worldwide burden of cancer in 2008: GLOBOCAN 2008. Int J Cancer 2010; 127: 2893 - 2917

2 Kinzler $K W$, Vogelstein $B$. Lessons from hereditary colorectal cancer. Cell 1996; 87: $159-170$

3 Fearon ER, Vogelstein B. A genetic model for colorectal tumorigenesis. Cell 1990; 61: 759-767

4 Jass JR. Classification of colorectal cancer based on correlation of clinical, morphological and molecular features. Histopathology 2007; 50: $113-130$

5 Leggett $B$, Whitehall $V$. Role of the serrated pathway in colorectal cancer pathogenesis. Gastroenterology 2010; 138: 2088-2100

6 Estrada RG, Spjut HJ. Hyperplastic polyps of the large bowel. Am J Surg Pathol 1980; 4: 127-133

7 Goldman H, Ming S, Hickock DF. Nature and significance of hyperplastic polyps of the human colon. Arch Pathol 1970; 89: 349-354

8 Jass JR, Baker K, Zlobec I et al. Advanced colorectal polyps with the molecular and morphological features of serrated polyps and adenomas: concept of a 'fusion' pathway to colorectal cancer. Histopathology 2006; 49: $121-131$

9 Otori K, Oda Y, Sugiyama K et al. High frequency of K-ras mutations in human colorectal hyperplastic polyps. Gut 1997; 40: 660-663

10 Tuppurainen K, Makinen JM, Junttila $O$ et al. Morphology and microsatellite instability in sporadic serrated and non-serrated colorectal cancer. J Pathol 2005; 207: 285 - 294

11 Boparai KS, van den Broek FJ, van Eeden S et al. Hyperplastic polyposis syndrome: a pilot study for the differentiation of polyps by using highresolution endoscopy, autofluorescence imaging, and narrow-band imaging. Gastrointest Endosc 2009; 70: 947-955

12 Hasegawa S, Mitsuyama K, Kawano $\mathrm{H}$ et al. Endoscopic discrimination of sessile serrated adenomas from other serrated lesions. Oncol Lett 2011; 2: 785-789 
13 Kimura T, Yamamoto E, Yamano HO et al. A novel pit pattern identifies the precursor of colorectal cancer derived from sessile serrated adenoma. Am J Gastroenterol 2012; 107: 460 - 469

14 Yano Y, Konishi K, Yamochi T et al. Clinicopathological and molecular features of colorectal serrated neoplasias with different mucosal crypt patterns. Am J Gastroenterol 2011; 106: 1351 - 1358

15 [Anonymous] The Paris endoscopic classification of superficial neoplastic lesions: esophagus, stomach, and colon: November 30 to December 1, 2002. Gastrointest Endosc 2003; 58: 3-43

16 Konishi K, Kaneko K, Kurahashi T et al. A comparison of magnifying and nonmagnifying colonoscopy for diagnosis of colorectal polyps: A prospective study. Gastrointest Endosc 2003; 57: 48 - 53

17 Kudo S, Tamura S, Nakajima T et al. Diagnosis of colorectal tumorous lesions by magnifying endoscopy. Gastrointest Endosc 1996; 44: 8-14

18 Snover D, Ahnen DJ, Burt RW et al. Serrated polyps of the colon and rectum and serrated ("hyperplastic") polyposis. In: et al. Bozman FT, Carneiro F, Hruban RH, eds. WHO classification of tumours. Pathology and genetics of tumours of the digestive system. 4 th edn. Berlin: SpringerVerlag; 2010

19 Clark SJ, Harrison J, Paul CL et al. High sensitivity mapping of methylated cytosines. Nucleic Acids Res 1994; 22: 2990 -2997
20 Konishi K, Shen L, Wang S et al. Rare CpG island methylator phenotype in ulcerative colitis-associated neoplasias. Gastroenterology 2007; 132: $1254-1260$

21 Issa JP. CpG island methylator phenotype in cancer. Nat Rev Cancer 2004; 4: 988 - 993

22 Shen L, Toyota M, Kondo $Y$ et al. Integrated genetic and epigenetic analysis identifies three different subclasses of colon cancer. Proc Natl Acad Sci USA 2007; 104: 18654-18659

23 Buda A, De Bona M, Dotti I et al. Prevalence of different subtypes of serrated polyps and risk of synchronous advanced colorectal neoplasia in average-risk population undergoing first-time colonoscopy. Clin Transl Gastroenterol 2012; 3: e6

24 Konishi K, Yamochi T, Makino R et al. Molecular differences between sporadic serrated and conventional colorectal adenomas. Clin Cancer Res 2004; 10: 3082 - 3090

25 Rex DK, Ahnen DJ, Baron JA et al. Serrated lesions of the colorectum: review and recommendations from an expert panel. Am J Gastroenterol 2012; 107: 1315-1329; quiz 1314, 1330

26 Tadepalli US, Feihel D, Miller KM et al. A morphologic analysis of sessile serrated polyps observed during routine colonoscopy (with video). Gastrointest Endosc 2011; 74: 1360 - 1368 